\title{
Editorial
}

Bundesgesundheitsbl 2016 59 :301-303

DOI 10.1007/s00103-016-2307-x

Online publiziert: 25. Januar 2016

(c) Springer-Verlag Berlin Heidelberg 2016

CrossMark
Iris Pigeot ${ }^{1} \cdot$ Michael Hummel $^{2}$

${ }^{1}$ Leibniz-Institut für Präventionsforschung und Epidemiologie - BIPS, Bremen, Deutschland

${ }^{2}$ Institut für Pathologie CBF - Molekulare Diagnostik, Campus Benjamin

Franklin, Charité - Universitätsmedizin Berlin, Berlin, Deutschland

\section{Biobanken - eine entscheidende Ressource für erfolgreiche biomedizinische Forschung}

Nicht erst seitdem das Time Magazine $2009^{1}$ das Biobanking zu einer der zehn wichtigsten Ideen gekürt hat, die die Welt verändern werden, ist dieses Thema verstärkt in den Fokus von Wissenschaftlern, Fördergebern, Industrie, Datenschützern, Juristen, Politikern und der Öffentlichkeit gerückt. Dass es sich dabei nicht nur um eine kurzfristige Modeerscheinung handelt, belegen zahlreiche neue Biobankstrukturen auf nationaler und internationaler Ebene, die einen zunehmenden Beitrag zur Generierung wissenschaftlicher Erkenntnisse vor allem auf dem Gebiet der personalisierten Medizin - einschließlich der Erforschung von Krankheitsursachen - leisten. Wissenschaftliche Ergebnisse auf Grundlage von Forschung mit Biomaterialien gab es auch schon vor dem Aufbau dieser Biobankstrukturen, doch es hat sich mehr und mehr gezeigt, dass die Biomaterialen häufig unzureichend beschrieben oder charakterisiert bzw. von unzureichender Qualität waren. Dies führte dazu, dass Ergebnisse oftmals in unabhängigen Studien nicht reproduziert werden konnten ${ }^{2}{ }^{3}$. Weiterführende Entwicklungen auf Grundlage der häufig hochrangig publizierten Daten führten in zahlreichen Fällen nicht zum Erfolg und verursachten

1 Park A. (2009) Biobanks. Time Magazine 173(11)

2 Begley CG, Ellis LM (2012) Drug development: raise standards for preclinical cancer research. Nature 483(7391):531-533

3 Prinz F, Schlange T, Asadullah K (2011) Believe it or not: how much can we rely on published data on potential drug targets? Nature Rev Drug Discov 10:712-713 enorme Kosten, die hätten vermieden werden können.

Nur Biobanken, die den hohen Anforderungen an die Qualität der Biomaterialien und der damit verbundenen klinischen und persönlichen Daten entsprechen, werden zukünftig als Partner in der biomedizinischen Forschung erfolgreich sein. Allerdings umfassen Biobanken, die diesem Leitgedanken folgen, weit mehr als den kontrollierten Betrieb von Tiefkühlschränken. Die häufig viel gepriesenen kleinen Sammlungen von Biomaterialien, z. B. im Rahmen von Doktorarbeiten angelegt, mögen auf den ersten Blick eine „sparsame“ Variante darstellen, sind aber bei genauerer Betrachtung in der Regel von unzureichender Qualität und entsprechen zum Teil nicht den erforderlichen datenschutzrechtlichen und ethischen Anforderungen. Insbesondere ist es fraglich, ob es ethisch verantwortlich ist, Patientenproben unter Bedingungen zu gewinnen, die eine spätere sinnvolle Nutzung für die Forschung nicht garantieren können. Um die Qualität auch von kleinen Sammlungen und damit ihre sinnvolle Nutzung garantieren zu können, ist es stattdessen empfehlenswert, auf existierende zentrale Biobankstrukturen wie z. B. die Zentrale Biomaterialbank der Charité und des Berliner Instituts für Gesundheitsforschung (biobank.charite. de) zurückzugreifen, die unter definierten und transparenten Rahmenbedingungen arbeiten.

Als Teil dieser Rahmenbedingungen müssen in einem ersten Schritt klare rechtliche und ethische Bedingungen zur Gewinnung von Biomaterialen festgelegt werden, die einen breiten Schutz der Patienten- und Probandenrechte umfassen. Voraussetzung ist eine für medizinische Laien verständliche Information und Aufklärung der Biomaterial spendenden Personen, die die jeweilige Personengruppe und Erkrankungssituation berücksichtigt. Damit wird die valide Grundlage für eine Einwilligung in eine Verwendung von Proben und Daten für die Forschung gelegt. Da jedoch bei Biomaterialien zum Zeitpunkt der Einlagerung nicht voll umfänglich bekannt ist, welche Forschungsprojekte zu einem späteren Zeitpunkt damit durchgeführt werden, spielen hier die Ethikkommissionen als Vertreter der Patienteninteressen eine entscheidende Rolle 4 . Dies ist bei zentralen Biobanken von besonderer Relevanz, da diese Biomaterialien primär nicht für die eigene Verwendung sammeln, verarbeiten und lagern, sondern für spätere wissenschaftliche Vorhaben. Daher müssen klare Regeln bestehen, unter welchen Voraussetzungen Proben und Daten einer Biomaterialbank für die Forschung weitergegeben werden können. Dazu gehört u. a., dass für jedes Vorhaben ein zustimmendes Ethikvotum eingeholt und eine datenschutzrechtliche Prüfung vorgenommen werden muss. Biomaterialien, die für Studien und Konsortien im Rahmen einer Dienstleistung verwaltet werden, unterliegen den Vergaberegeln

\footnotetext{
4 Arbeitskreis Medizinischer EthikKommissionen in der Bundesrepublik Deutschland e. V.; http://www.ak-med-ethik-komm.de/ index.php/de/biobanken
} 
der jeweiligen Studie. Für Biomaterialien und Daten aus dem klinischen Kontext, die ohne konkrete Zweckbestimmung für zukünftige Forschungsprojekte gesammelt wurden, sind die Biobanken selbst an der Entscheidung über die Weitergabe beteiligt. Dabei läuft die Entscheidung über die Weitergabe in aller Regel über ein geeignet besetztes Vergabekomitee.

Als zentralem Ausgangspunkt für den Betrieb von Biobanken widmen sich die ersten drei Beiträge dieses Hefts daher dem oben skizzierten Themenkreis. Zunächst diskutieren J. Taupitz und M. Schneider das Spannungsfeld, das sich aus dem Persönlichkeitsrecht der Spender und der Wissenschaftsfreiheit der Forscher ableitet und das es in jedem Forschungsprojekt aufzulösen gilt. Sie legen zu diesem Zweck den aktuellen Stand der Diskussion dar und geben Hinweise für die Praxis. Der Beitrag von R. Jahns widmet sich ebenfalls der Abwägung zwischen der Wahrung der Forschungsfreiheit und den Interessen der Spender, die bereits bei dem Aufbau und natürlich bei dem späteren Nutzen von Biobanken berücksichtigt werden müssen. Diese Abwägung muss sich auch in wohl durchdachten und transparenten Nutzungskonzepten niederschlagen, die bei humanen Biomaterialien von besonderer Brisanz sind, da diese wertvollen Materialien nur begrenzt zur Verfügung stehen und deren Nutzung zudem, wie in den obigen zwei Beiträgen ausgeführt, strengen Regularien unterliegt. Dieses wichtige Thema behandeln R. Siddiqui und S.C. Semler daher in einem eigenen Beitrag.

Eine garantiert exzellente Qualität von Biomaterialien ist eine der wichtigsten Triebfedern für den Aufbau von professionellen Biobankstrukturen, die als Dienstleister für alle Arten von Biomaterialsammlungen dienen. Nur so lassen sich einheitliche und gleichbleibende Qualitätsmerkmale garantieren, die auch biobankübergreifende Forschungsprojekte deutlich erleichtern bzw. erst ermöglichen ${ }^{5}$. Die Definition von Quali-

\footnotetext{
5 Deutsches Konsortium für Translationale Krebsforschung (DKTK); http://www.dkfz.de/ de/dktk/
}

tät bleibt ein mit viel Leidenschaft diskutiertes Thema. Je nach Probenart und je nach geplanter methodischer Verwendung der Proben sind die Anforderungen an die Qualität des Biomaterials und damit an die Biobankprozesse und die Maßnahmen zur Qualitätssicherung jedoch sehr unterschiedlich, wie in dem Beitrag von E. Herpel, S. Schmitt und M. Kiehntopf am Beispiel von Flüssig- und Gewebeproben deutlich wird. Die Etablierung von zentralen Biobankstrukturen ermöglicht hier die Einhaltung von gemeinsam definierten Qualitätsstandards für vergleichbare Sammlungen und deren Anwendungen im großen Maßstab und über einen langen Zeitraum ${ }^{6}$. I. Bernemann und Kollegen beleuchten daher in ihrem Beitrag im Detail die Anforderungen an zentrale Biobanken als Grundlage für die medizinische Forschung, während sich M. Thomas in ihrem Beitrag speziell der Bedeutung von Biobanken für die klinische Forschung und damit für eine effektivere und effizientere Arzneimittelversorgung widmet.

Der Nutzen von Biobanken als Forschungsressource wird noch einmal deutlich vergrößert, wenn man Biomaterialen mit klinischen Daten, allgemeinen Gesundheitsdaten, Informationen $\mathrm{zu}$ den Lebensgewohnheiten oder mit molekularen Analysedaten verknüpft. Durch die Zusammenführung dieser Daten entsteht ein umfassendes Bild des Individuums, das in falschen Händen jedoch auch missbraucht werden könnte. Um die Daten in einer Biobank bestmöglich zu schützen, sind effektive Datenschutzmaßnahmen unerlässlich. Hierfür gibt es hervorragende Konzepte, die über die vergangenen Jahre insbesondere durch die Arbeitsgruppe „Datenschutz" an der TMF entwickelt wurden und ständig verbessert werden ${ }^{7}$. Die Anwendung dieser Datenschutzmaßnahmen bietet einen zuverlässigen Schutz sämtlicher Daten von Probanden, die ihre

\footnotetext{
6 Technologie und Methodenplattform für die vernetzte medizinische Forschung e.V. (TMF); http://www.tmf-ev.de/Produkte/Materialien_ LeitlinieDatenqualitaet.aspx

7 TMF: Arbeitsgruppe Datenschutz; http:// www.tmf-ev.de/Arbeitsgruppen_Foren/AGDS. aspx
}

Proben und Daten einer Biobank anvertrauen.

Allerdings muss bei der Verknüpfung verschiedener Datenquellen eine Qualitätssicherung jeder Quelle erfolgen. So sind insbesondere die Daten, die im Rahmen von Diagnostik und Therapie erhoben werden, für klinisch orientierte Biobanken von größter Wichtigkeit. Diese Daten stehen jedoch nur in sehr unterschiedlichem Umfang und unterschiedlicher Qualität zur Verfügung - und dies ist durch die Biobanken selbst nur bedingt beeinflussbar. Daher müssen zukünftig Lösungen gefunden werden, um klinische Daten möglichst umfassend und strukturiert mit Biomaterialien verknüpfen zu können und diese auch für Forschungsprojekte anbieten zu können. Im Rahmen von epidemiologischen oder studienbezogenen Biobanken, in denen Daten projektspezifisch erhoben werden, lassen sich naturgemäß homogenere Daten mit hohen Qualitätsstandards erheben als in einem heterogenen klinischen Umfeld. Dies wird auch deutlich in dem Beitrag von H.E. Wichmann und Kollegen, in dem das große Potenzial der Nationalen Kohorte als Forschungsinfrastruktur für Deutschland aufgezeigt wird, das insbesondere aus der Verknüpfung von hochwertigen Bioproben und detaillierten Informationen aus medizinischen Untersuchungen und Befragungen resultiert. Das Prinzip dieser großen bevölkerungsbezogenen epidemiologischen Studie ist vergleichbar zu dem der UK-Biobank, die als ein weiteres Beispiel für das große Forschungspotenzial von Biobanken, verknüpft mit einer eingehenden Phänotypisierung der Studienteilnehmer, in dem Beitrag von A. Trehearne vorgestellt wird. Ein weiteres beeindruckendes Beispiel für eine nationale Probenbank ist die Umweltprobenbank des Bundes, die bereits seit 1979 betrieben wird und deren Ziel es ist, den Schutz von Mensch und Umwelt vor schädlichen Umwelteinflüssen sicherzustellen, wie C. Schröter-Kermani, A. Gies und M. Kolossa-Gehring in ihrem Beitrag herausarbeiten.

Eine weitere Herausforderung an Biobanken ergibt sich durch die zunehmende Internationalisierung von Forschungsprojekten. Um Deutschland eine zentrale Rolle in der internationalen Zusammen- 
arbeit zu ermöglichen, müssen sich die nationalen Biobanken entsprechend international - insbesondere europäisch - ausrichten. Dies setzt sowohl auf nationaler als auch auf internationaler Ebene die Verwendung gleicher Begrifflichkeiten voraus oder zumindest die Möglichkeit, unterschiedliche Ontologien über ein „Mapping" miteinander kompatibel zu machen ${ }^{8}$. Entsprechende Aktivitäten, die M. Lablaus und Kollegen in ihrem Beitrag ausführlich beschreiben, werden derzeit im Rahmen des "Common Service IT" des pan-europäischen Biobanken-Netzwerks BBMRI-ERIC durchgeführt ${ }^{9}$. Die Bedeutung solcher Netzwerke wird in $\mathrm{Zu}$ kunft noch erheblich zunehmen, da die $\mathrm{Zu}$ sammenstellung von ausreichend großen Probenkollektiven mit ausreichender Probenqualität und umfangreichen Daten nicht immer von einer einzigen Biobank geleistet werden kann. BBMRI-ERIC soll daher auch eine Schnittstelle für einen gerecht geregelten Zugang zu qualitätskontrollierten humanbiologischen Proben und deren verbundenen biomedizinischen und molekularen Daten bilden, wie M. Mayrhofer und Kollegen in ihrem Beitrag darlegen. In Ergänzung zu BBMRIERIC stellen K. Kuhn und Kollegen in ihrem Beitrag eine weitere europäische Initiative vor, die es sich zum Ziel gesetzt hat, Biobanken großer europäischer Kohorten zu vernetzen, wodurch eine länderübergreifende Forschung zu wichtigen Krankheitsfeldern und zur Gesundheitsvorsorge ermöglicht werden soll.

Der Deutsche Biobankknoten (German Biobank Node, $\mathrm{GBN}^{10}$ ) ist sowohl auf nationaler als auch auf europäischer Ebene aktiv. National dient GBN als zentrale Kontakt- und Vermittlungsstelle für alle Biobankbelange. Auf europäischer Ebene hat GBN (BBMRI.de) die Aufgabe, die deutschen Biobankinteressen $\mathrm{zu}$ vertreten und die europäische $\mathrm{Zu}$ sammenarbeit im Rahmen von BBMRIERIC mitzugestalten. Damit sind die deutschen Biobanken über GBN/BBMRI.

\footnotetext{
8 Mate S, Köpcke F, Toddenroth D, Martin M, Prokosch H-U, Bürkle T, Ganslandt T (2015) Ontology-based data-integration between clinical and research systems. PLoS One 10(1): e0116656

9 BBMRI-ERIC; http://bbmri-eric.eu

10 German Biobank Node; http://bbmri.de
}

de maßgeblich an der Gestaltung von europäischen Qualitätskriterien, ethischen und rechtlichen Strukturen sowie am Aufbau eines Biobanken-IT-Netzwerkes beteiligt, das zukünftig eine Biobank-übergreifende Abfrage nach Biomaterialien und deren Daten über europäische Grenzen hinweg ermöglichen wird. Auf der anderen Seite spiegelt GBN/BBMRI. de auch innovative Modelle aus anderen europäischen Ländern in die deutsche Biobank-Community zurück (z. B. http://www.biopankki.fi/en/suomalaisetbiopankit/), die mithelfen können, bestehende strukturelle Nachteile zu überwinden, um auch langfristig auf dem internationalen Parkett mitzuspielen. Aufgrund der föderalen Struktur, dem Fehlen von zentralen Personenidentifikatoren und unzureichenden gesetzlichen Rahmenbedingungen zur Verwendung von Versorgungsdaten für die Forschung hat Deutschland einen Wettbewerbsnachteil, der sich auch durch finanzielle Investitionen alleine nicht ausgleichen lässt. Hier auf Veränderungen hinzuwirken, muss oberstes Ziel von GBN zusammen mit den Biobankenbetreibern und -nutzern sein.

Jedoch macht der Aufbau zentraler Biobanken nur Sinn, wenn die damit geschaffenen Strukturen langfristig angelegt werden. Als Ausblick befassen sich R. Kirsten und Kollegen in dem letzten Themenbeitrag dieses Hefts daher mit Fragen zur Sicherung der Nachhaltigkeit von Biobanken. Dabei spielt die Finanzierung eine zentrale Rolle. Obwohl zentrale Biobanken wesentlich kostengünstiger arbeiten können als viele kleine separate Biobanken, ist der initiale Aufbau und laufende Betrieb mit erheblichen Gesamtkosten verbunden. Für zentrale Biobankstrukturen sollte die betreibende Einrichtung eine Grundfinanzierung bereitstellen, die den Betrieb auch personell garantiert. Darüber hinaus muss ein Teil der Kosten durch Aufwandsentschädigungen (z. B. für Dienstleistungen im Rahmen von Drittmittelprojekten) für die Leistungen der Biobank kompensiert werden. Weitere Mittel sollten im Rahmen von Projektförderungen eingeworben werden können, die für technische und inhaltliche Weiterentwicklungen sowie den Aufbau von
Netzwerken auf zielgerichtete Biobankförderungen ausgeschrieben werden. Eine wesentliche Grundlage der Finanzierung von Biobanken sind transparente Kostenmodelle für die Leistungen von Biobanken. Damit können auch Zusammenstellungen von Kollektiven über Biobanken deutlich einfacher realisiert werden.

Die Biobanken in Deutschland sind mit ihren Aktivitäten auf dem richtigen Weg. Durch die Kommunikation innerhalb der deutschen Biobanken, die fortschreitende Konsolidierung der bisher fragmentierten Biobanklandschaft, die Harmonisierung von Prozessen und Strukturen sowie die nationale und internationale Vernetzung wird eine solide Grundlage für eine erfolgreiche biomedizinische Forschung in Deutschland geschaffen. Damit werden zukünftig Forschungsdaten zusammen mit Biomaterialen ein zuverlässiger Ausgangspunkt sein, um Krankheiten besser zu verstehen und gezielter bekämpfen zu können.

Wir hoffen, dass wir Sie auf die Beiträge zu diesem Thema neugierig gemacht haben, und wünschen Ihnen eine interessante Lektüre.

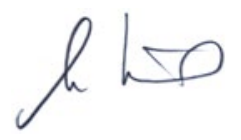

Ihr Michael Hummel

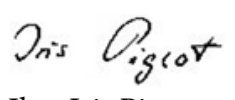

Ihre Iris Pigeot

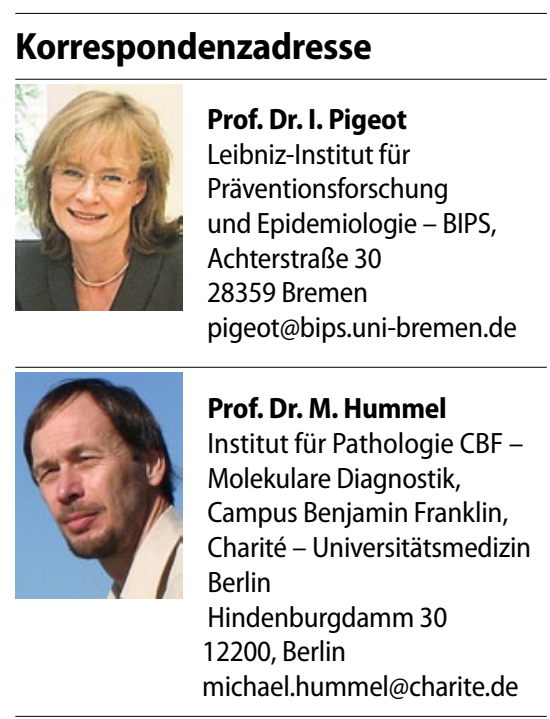

